\title{
Strategische Überlegungen bei mit spinaler Ischämie assoziertem intramuralem Hämatom
}

\section{Anamnese}

Ein bisher gesunder 60-jähriger Patient wurde unter der Verdachtsdiagnose eines IMH der Aorta descendens in die gefäßchirurgische Klinik unseres Hauses übernommen. Der Patient hatte sich zuvor auswärtig mit starken Thoraxschmerzen sowie bereits schwerem motorischen Defizit der unteren Extremität vorgestellt. An Nebenerkrankungen und vaskulären Risikofaktoren bestanden eine bisher unbehandelte arterielle Hypertonie sowie ein Nikotinabusus von 45 pack-years.

\section{Befund und Diagnose}

Bei Aufnahme bestand eine komplette Parese beider Beine bei erhaltener Sensibilität. Der Pulsstatus war unauffällig, der ABI lag beidseits im Normbereich. Die Computertomographie-Angiographie (CTA) der gesamten Aorta (Schichtdicke: $1 \mathrm{~mm}$ ) bestätigte die Verdachtsdiagnose eines nach der linken A. subclavia (LSA) beginnenden IMH, Typ Stanford B. Der Befund erstreckte sich nach distal bis an die A. mesenterica inferior und wies zwei kleine, aktiv über die Interkostalarterien $T_{5}$ und $\mathrm{T} 6$ perfundierte Einblutungen auf. Es bestand kein Hinweis auf ein

Infobox 1 Interventionskriterien bei Intramuralem Hämatom (Тур B)

- Größenprogredienz (Aortendurchmesser/ Hämatomdurchmesser)

- Ausbildung einer Dissektion

- Offene/gedeckte Ruptur

- Persistierender/wiederkehrender Schmerz

- Therapierefraktärer Hypertonus
Entry. Die thorakalen und lumbalen Segmentarterien zeigten sich bis auf die Interkostalen $\mathrm{T}_{3}$ und $\mathrm{T}_{4}$ regelrecht kontrastiert (- Abb. 1a). In einer spinalen Magnetresonanztomographie (MRT) stellte sich in der T2-Wichtung eine zentral symmetrische Signalanhebung in Höhe des Conus medullaris im Sinne einer Rückenmarkischämie mit Rückenmarködem dar (• Abb. 1b)

\section{Therapie und Verlauf}

Bereits extern war nach Rücksprache aufgrund der rasch progredienten Lähmungserscheinungen eine lumbale Liquordrainage (LD) initiiert worden. Liquordruck und mittlerer arterieller Druck (MAD) wurden auf Zielwerte unter $15 \mathrm{mmHg}$ bzw. unter $80 \mathrm{mmHg}$ eingestellt. Eine nach erneutem thorakalen Schmerzereignis durchgeführte KontrollCTA zeigte eine Progression des Befunds im Sinne eines neu aufgetretenen Dissektionsentrys in Höhe Th 8 ( $\bullet$ Abb. 2a). Daraufhin erfolgte die transfemorale Implantation zweier thorakaler Stentgrafts (C-TAG ${ }^{\circledR}$, W.L. Gore \& Associates, Inc., Arizona, USA, Maße: 26-21-10o und 2121-100). Die angiographische Kontrolle zeigte eine erfolgreiche Ausschaltung mit kompletter Abdeckung des ventralen Entrys (- Abb. 2b). Das gestentete Segment reichte von distal der LSA bis zu Th 9 (- Abb. 2c). Im Verlauf wies der Patient die persistierenden klinischen Symptome eines A.-spinalis-anterior-Syndroms auf.

Die CTA am 6. postoperativen Tag zeigte umschriebene intramurale KM-Enhancements im Sinne eines IMH über die retrograd perfundierten Interkostalgefä- ße T5 und T6 (• Abb. 2c). Die LD wurde nach 7 Tagen beendet. Bei Entlassung lag noch immer eine komplette Parese der unteren Extremität vor.

\section{Diskussion}

Die vorgestellte Kasuistik beschreibt den seltenen Fall einer spinalen Ischämie als Primärsymptom eines intramuralen Hämatoms der Aorta descendens. Es bestand der therapeutische Konflikt zwischen einer - bei progredienter IMH indizierten - langstreckigen endovaskulären Versorgung im Sinne der Rupturprophylaxe und dem dadurch bedingten erhöhten Risiko einer irreversiblen Paraplegie.

Der natürliche Verlauf des IMH ist dynamisch. Der Übergang in eine klassische Dissektion oder (gedeckte) Ruptur ist derzeit nicht prognostizierbar. Als Primärsymptom gilt der Thoraxschmerz. In Diagnostik und Follow-up kommt der Bildgebung mittels Dünnschicht-CTA und multiplanarer Rekonstruktion eine entscheidende Rolle zu. Die Einteilung des IMH orientiert sich an der Stanford-Klassifikation für Dissektionen [10]. Auch die Therapie des IMH stützt sich weitestgehend auf die Daten zur Behandlung der klassischen Aortendissektion, wonach das Typ A IMH (39,7\% aller IMH) im Sinne eines Notfalleingriffs sofort kardiochirurgisch versorgt werden sollte [7]. Bei Typ B IMH (60,3 \%) ist bei unkomplizertem stabilen Befund ein primär konservatives Procedere unter Beachtung der potenziellen Dynamik der Läsion gerechtfertigt [3]. Als grundsätzliche Indikationen zur endovaskulären Versorgung von Typ B IMHs gelten die in • Info- 


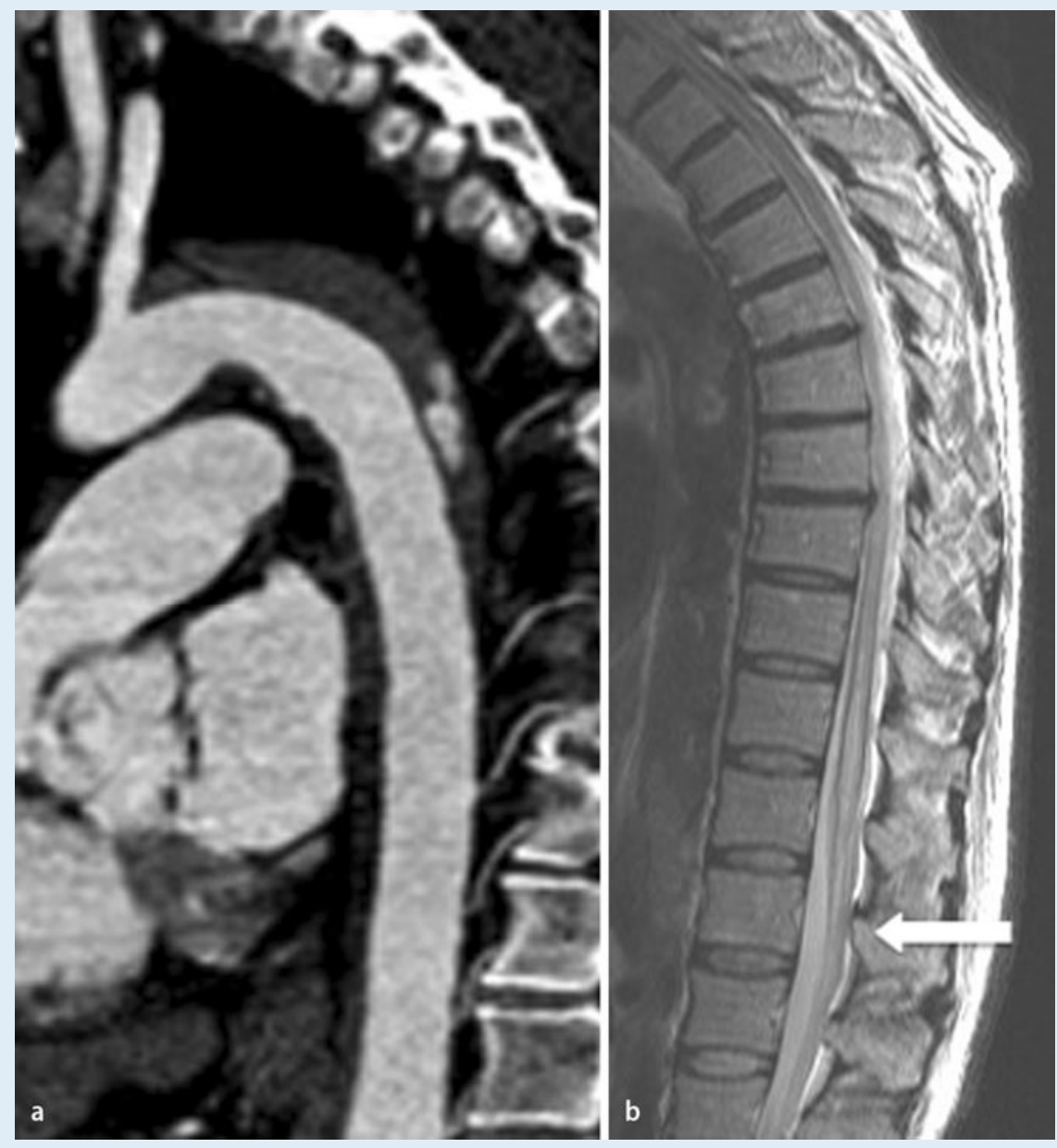

Abb. $1 \Delta$ a Die CT-Angiographie bei Aufnahme zeigte zwei kleine Einblutungen im Bereich der Interkostalen T5 und T6. Unterhalb davon lag eine regelrechte Kontrastierung der Segmentarterien vor. b Auftreibung des kaudalen Rückenmarks bzw. des Conus medullaris in Höhe der unteren Brust-, und oberen Lendenwirbelsäule (weißer Pfeil). Die T2-gewichteten sagittalen MRT-Sequenzen zeigten ein zentral hyperintenses Signal im Sinne eines raumfordernden Rückenmarködems
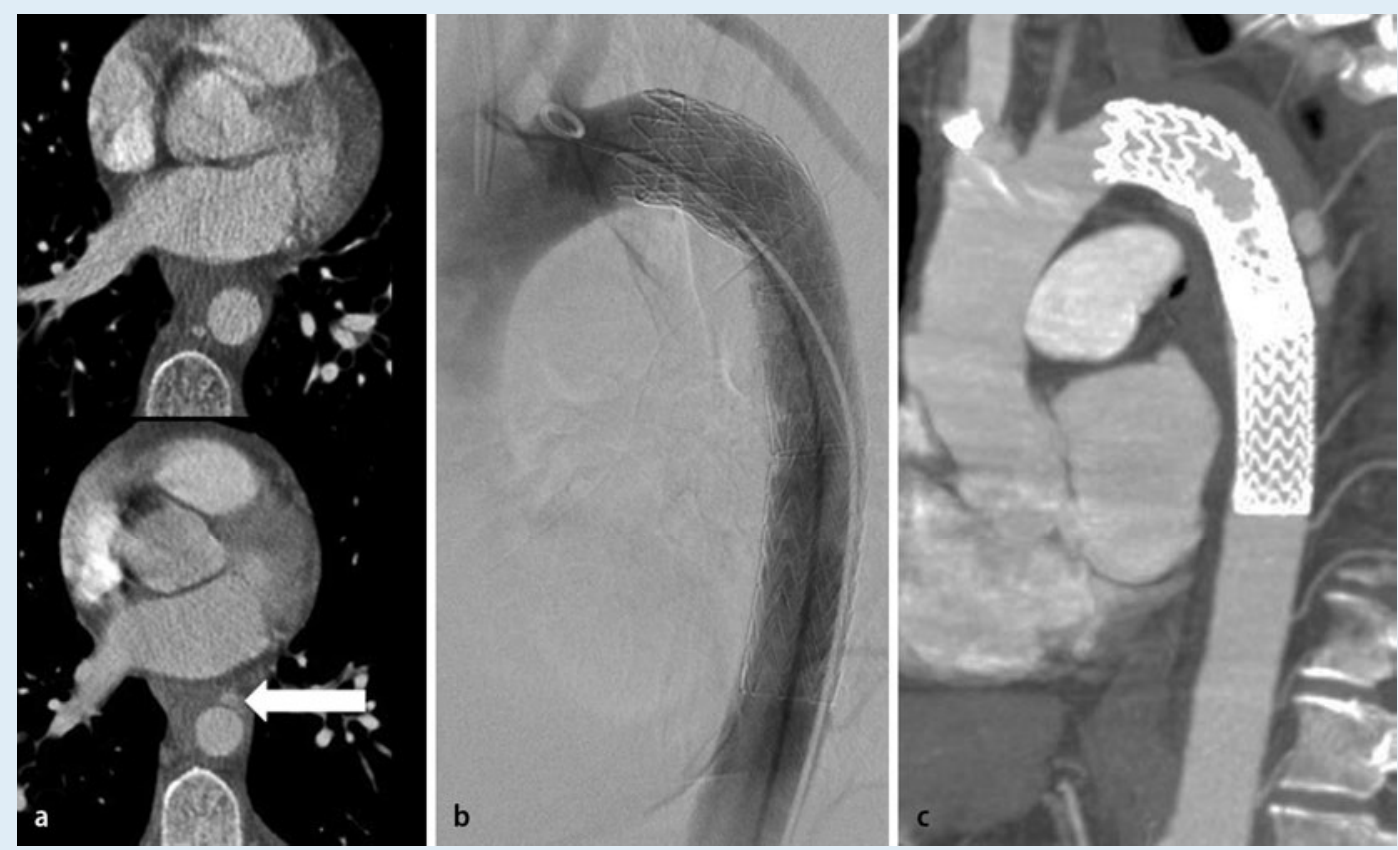

Abb. $2<$ a Gegenüberstellung der Aufnahme-CT-Angiographie (oben) mit der präoperativen Verlaufs-CTAngiographie (unten). Es zeigte sich eine neu aufgetretene ventrale Einblutung (weißer Pfeil) auf Höhe des 9 . Brustwirbelkörpers im Sinne einer Progression des IMH. b Die intraoperative Angiographie zeigt eine erfolgreiche Ausschaltung des IMH. c Auch nach TEVAR zeigten sich die beschriebenen Einblutungen retrograd perfundiert 
M.S. Bischoff $\cdot$ A.S. Peters $\cdot$ D. Kotelis · D. Böckler · A. Hyhlik-Dürr Strategische Überlegungen bei mit spinaler Ischämie assoziertem intramuralem Hämatom

\section{Zusammenfassung}

Das intramurale Hämatom (IMH) zählt mit dem penetrierenden Aortenulkus und der klassischen Aortendissektion zur Entität des akuten Aortensyndroms. Typischerweise präsentiert sich das IMH mit akutem Brust- oder Rückenschmerz. Eine Durchblutungsstörung des Rückenmarks als Initialsymptom eines IMH ist hingegen selten. Wir beschreiben anhand eines Fallberichts das diagnostische Vorgehen und diskutieren therapeutische Überlegungen bei einem mit spinaler Ischämie assozierten IMH.

Schlüsselwörter

Intramurales Hämatom · Spinale Ischämie Aortendissektion · Paraplegie/Paraparese

\section{Strategic considerations \\ for intramural hematoma assocated with spinal ischemia}

\section{Abstract}

Intramural hematoma (IMH) forms - along with classic aortic dissection and penetrating aortic ulcer - the entity of acute aortic syndrome. Whereas thoracic pain is a common symptom in these patients, an association of IMH with spinal cord ischemia has been rarely described. We present a case of lower limb paraparesis in a patient with IMH type B. General considerations regarding IMH evaluation and treatment are discussed in the face of spinal cord protection strategies.

\section{Keywords}

Inramural hematoma $\cdot$ Spinal cord ischemia Aortic dissection · Paraplegia/paraparesis box 1 aufgeführten komplizierten Verläufe. Ziel einer endovaskulären Versorgung ist der zuverlässige Ausschluss des $\mathrm{IMH}$, bei Bogenbeteiligung ggf. im Rahmen eines Hybridverfahrens [4]. Im Falle des vorgestellten Patienten zeigte sich der Befund in der CTA zunächst stabil. Auf die prinzipiell bei spinaler Ischämie obligate Steigerung des MAD auf Werte um $90 \mathrm{mmHg}$ im Sinne einer spinalen Perfusionssteigerung wurde unmittelbar bei Aufnahme verzichtet, um die Progredienz des Befunds zu vermeiden [1]. Die Indikation zur TEVAR erfolgte aufgrund des bei Befundprogredienz zunehmenden Rupturrisikos.

Eine direkte endovaskuläre Versorgung wäre mit einer unwiederbringlichen Okklusion zusätzlicher, noch regulär perfundierter, Segmentarterien einhergegangen. Dieses Vorgehen hätte unter Umständen die Chance auf eine Reversibilität des neurologischen Defizits weiter vermindert. Nach Endograftplatzierung konnte der MAD ohne Gefahr der persistierenden Ruptur zur Verbesserung der Spinalperfusion angehoben werden. Aufgrund der anatomischen Lage des IMH war es möglich, den proximalsten der drei Endografts distal der LSA zu platzieren und somit den proximalen Einstrom in das spinale Kollateralnetzwerk zu erhalten [1]. Anhand von EUROSTAR-Daten konnte gezeigt werden, dass ein Überstenten der LSA mit einem erhöhten Paraplegierisiko einhergeht [2]. Wenn eine Schonung des Gefäßes aus technischen oder anatomischen Gründen nicht möglich ist, sollte daher eine Revaskularisation der LSA erfolgen. Dies gilt insbesondere für den aortal voroperierten Patienten sowie in Fällen, bei denen eine langstreckige endovaskuläre Versorgung angezeigt ist [5].

In der Literatur der letzten 10 Jahre finden sich 2 beschriebene Fälle, in denen ein isoliertes IMH primär mit einer Rückenmarkischämie assoziiert war [8]. Ohmi et al. berichteten über ein mit Paraplegie vergesellschaftetes Typ A IMH. Sowohl das neurologische Defizit als auch der radiologische Befund bildeten sich unter konservativer Therapie zurück. Motoyoshi et al. beschrieben 2003 den Fall eines 46-jährigen Patienten mit links betonter Hemiparese bei Typ A IMH. Durch Senkung des intraspinalen Drucks mittels LD kam es im Verlauf zur vollständigen Rückbildung der Symptomatik [6]. Dem kontrollierten Ablassen von Liquor kommt eine entscheidende Rolle in der Prävention der Rückenmarkschädigung in der Aortenchirurgie zu. Durch Senkung des intrathekalen Drucks (Drainage von ca. 10$15 \mathrm{ml} / \mathrm{h}$; Zielwert: 10-15 $\mathrm{mmHg}$ ) kann die Rückenmarkperfusion verbessert und die ödembedingte Drucksteigerung im Spinalkanal abgemindert werden [1]. Eine kontinuierliche Druckmessung ist hierbei angezeigt, da eine überschießende Senkung des intrathekalen Drucks zu intrakranieller Hypotonie und zerebralen Blutungen führen kann. Der Nutzen einer medikamentösen Therapie des ischämiebedingten Rückenmarködems mit Steroiden (typischerweise Methylprednisolon $30 \mathrm{mg} / \mathrm{kg}$ i.v.) ist nicht belegt [9]. Im vorliegenden Fall war das neurologische Defizit trotz unmittelbarer Anlage einer LD nicht reversibel. Die Synopsis aus Klinik und MRT-Befund lässt darauf schließen, dass es zu einem radikulären Territorialinfarkt kam.

\section{Fazit für die Praxis}

Die Indikationsstellung zur endovaskulären Versorgung eines mit Paraplegie assoziierten IMH Typ B ist erschwert durch den Konflikt des bei langstreckigem Stentgrafting zusätzlich iatrogen erhöhten Risikos einer irreversiblen Spinalischämie. Bei Befundprogredienz ist die TEVAR die Methode der Wahl im Sinne der Lebensrettung. Wann immer möglich, sollte vorgeschaltet eine Revaskularisation der LSA erfolgen. Der Liquordrainage kommt in diesem Zusammenhang sowohl therapeutische als auch prophylaktische Bedeutung der spinalen Ischämie zu.

\section{Korrespondenzadresse Dr. M.S. Bischoff

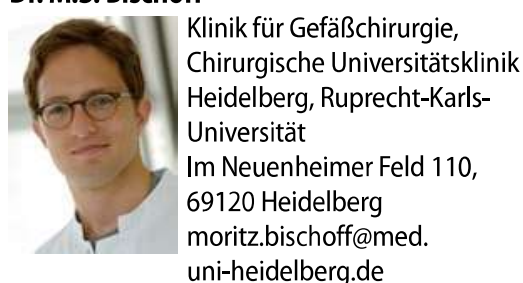

Interessenkonflikt. Der korrespondierende Autor gibt an, dass kein Interessenkonflikt besteht. 


\section{Literatur}

1. Bischoff MS, Di Luozzo G, Griepp EB et al (2011) Spinal Cord Preservation in Thoracoabdominal Aneurysm Repair. Perspect Vasc Surg Endovasc Ther [Epub ahead of print] doi:10.1177/1531003511400622

2. Buth J, Harris PL, Hobo R et al (2007) Neurologic complications associated with endovascular repair of thoracic aortic pathology: Incidence and risk factors. J Vasc Surg 46:1103-1110

3. Evangelista A, Mukherjee D, Mehta RH et al (2005) Acute intramural hematoma of the aorta: a mystery in evolution. Circulation 111:1063-1070

4. Geisbusch P, Kotelis D, Muller-Eschner M et al (2011) Complications after aortic arch hybrid repair. J Vasc Surg 53:935-941

5. Kotelis D, Geisbusch P, Hinz U et al (2009) Short and midterm results after left subclavian artery coverage during endovascular repair of the thoracic aorta. J Vasc Surg 50:1285-1292

6. Motoyoshi N, Komatsu T, Moizumi Y et al (2003) Atypical paraplegia after aortic intramural hematoma. J Thorac Cardiovasc Surg 125:409-410

7. Nienaber CA, Kodolitsch Y von, Petersen B et al

(1995) Intramural hemorrhage of the thoracic aorta. Diagnostic and therapeutic implications. Circulation 92:1465-1472

8. Ohmi M, Shibuya T, Kawamoto S et al (2003) Spinal cord ischemia complicated with acute aortic dissection and intramural hematoma; report of two cases. Kyobu Geka 56:473-478

9. Sinha AC, Cheung AT (2010) Spinal cord protection and thoracic aortic surgery. Curr Opin Anaesthesiol 23:95-102

10. Sundt TM (2007) Intramural hematoma and penetrating atherosclerotic ulcer of the aorta. Ann Thorac Surg 83:835-841 